\title{
COSMIC BACKGROUND X-RAYS PRODUCED BY INTERGALACTIC INNERBREMSSTRAHLUNG
}

\author{
SATIO HAYAKAWA \\ Dept. of Physics, Nagoya University, Nagoya, Japan
}

It has been found difficult to explain both the absolute intensity and energy spectrum of the background component of cosmic X-rays in terms of a superposition of X-ray sources in distant galaxies [1] and of the inverse Compton collisions of metagalactic electrons with microwave photons [2]. Although the absolute intensity could be obtained by choosing suitable values of parameters which are not well known as yet, the gradual bending of the X-ray spectrum around $30 \mathrm{keV}$ provides a critical test of theories. In this respect innerbremsstrahlung of intergalactic protons colliding with electrons is a candidate of background X-rays, since the X-ray spectrum is closely related to the proton spectrum and the latter usually bends in the non-relativistic region.

Innerbremsstrahlung is associated with the production of knock-on electrons by protons. The latter is a part of the ionization process which is responsible for heating the intergalactic medium [3]. Thus the background component of cosmic X-rays may provide a means of studying the thermal history of the universe.

The proton spectrum may bend due either to ionization energy loss or to an energy dependence of the acceleration rate or to both. If the former is the case, the spectrum bends at about [4]*

$$
E_{c}=\left(\frac{3 a n_{0} t_{0}}{2 M c^{2}}\right)^{2 / 3} m c^{2} \approx 6.5 \mathrm{keV},
$$

where $a \approx 3 \times 10^{-7} \mathrm{eV} \mathrm{cm}^{3} \mathrm{sec}^{-1}$ is an ionization energy loss rate, $n_{0}$ the present electron density, $t_{0}$ the present age of the universe, and $M$ and $m$ the proton and electron masses, respectively. The numerical value is obtained for $n_{0}=10^{-5} \mathrm{~cm}^{-3}$ and $t_{0}=3 \times 10^{17} \mathrm{sec}$; this is too small compared with the observed bending energy of about $30 \mathrm{keV}$. The acceleration at a rate proportional to total energy gives a power law in the relativistic region, but the kinetic energy spectrum in the non-relativistic region becomes less steep as energy decreases. Although we do not know a precise shape of the spectrum, unless detail of the acceleration process is known, it is quite likely that the proton spectrum in kinetic energy changes its slope at several tens of $\mathrm{MeV}$. This may be transferred to the X-ray spectrum which also changes its slope as observed.

If the cosmic ray production rate varies with cosmic age as $t^{-2}$, as expected from radio star count, and the production started at the redshift parameter $z=10$, the absolute intensity of background cosmic rays can be explained for the present value

* The X-ray energy relative to the proton energy given in [4] should be reduced by a factor of 4 . 
of the cosmic ray energy density times matter density of about $10^{-20} \mathrm{erg} \mathrm{cm}^{-6}$. For $n_{0}=10^{5} \mathrm{~cm}^{-3}$, the cosmic ray energy density of $10^{-15} \mathrm{erg} \mathrm{cm}^{-3}$ is consistent with that given in [3].

\section{References}

[1] Fujimoto, M., Hayakawa, S., and Kato, T.: 1969, Astrophys. Space Sci. 4, 64.

[2] Fukui, M. and Hayakawa, S.: 1969, Progr. Theor. Phys. 42, 119.

[3] Ginzburg, V. L. and Ozernoi, L. M.: 1966, Soviet Astron.-AJ 9, 726.

[4] Hayakawa, S.: 1969, Prog. Theor. Phys. 41, 1592. 